\title{
Erratum: Simplified approach to the repulsive Bose gas from low to high densities and its numerical accuracy [Phys. Rev. A 103, 053309 (2021)]
}

\author{
Eric A. Carlen, Markus Holzmann, Ian Jauslin @ ${ }^{\text {, }}$, and Elliott H. Lieb
}

(Received 4 October 2021; published 25 October 2021)

DOI: 10.1103/PhysRevA.104.049904

We have found an error in in the numerical calculation of the two-point correlations: the corrected Fig. 5 (below) happily shows a much better agreement between our equations and quantum Monte Carlo calculations. In addition, we have found a typographical error in the caption of Fig. 5: the potential is $16 e^{-|\mathbf{x}|}$ and not $e^{-|\mathbf{x}|}$.

These updates do not change the conclusions of the paper. In fact, the updated graph does not exhibit the unphysical numerical artifact we noted in our paper and strengthens the argument that the simplified approach captures some of the physics of the many-body Bose gas.

In the erroneous figure, the two-point correlation came out negative for small $|\mathbf{x}|$, which we suspected to be a numerical artifact (see the last sentence of Sec. III C), and this has now been shown to be the case: after fixing the numerical issue, the two-point correlation comes out positive as it should be.

The updated result for the medium equation is also much closer to the big equation than in our previous paper, and it exhibits the same bump, which was an unexpected find and is yet to be fully understood. This is further evidence that the medium equation reproduces some of the nontrivial physical behavior of the Bose gas.

Since the publication of this paper, the code for the numerical computations in the paper has been made available online in Ref. [1].
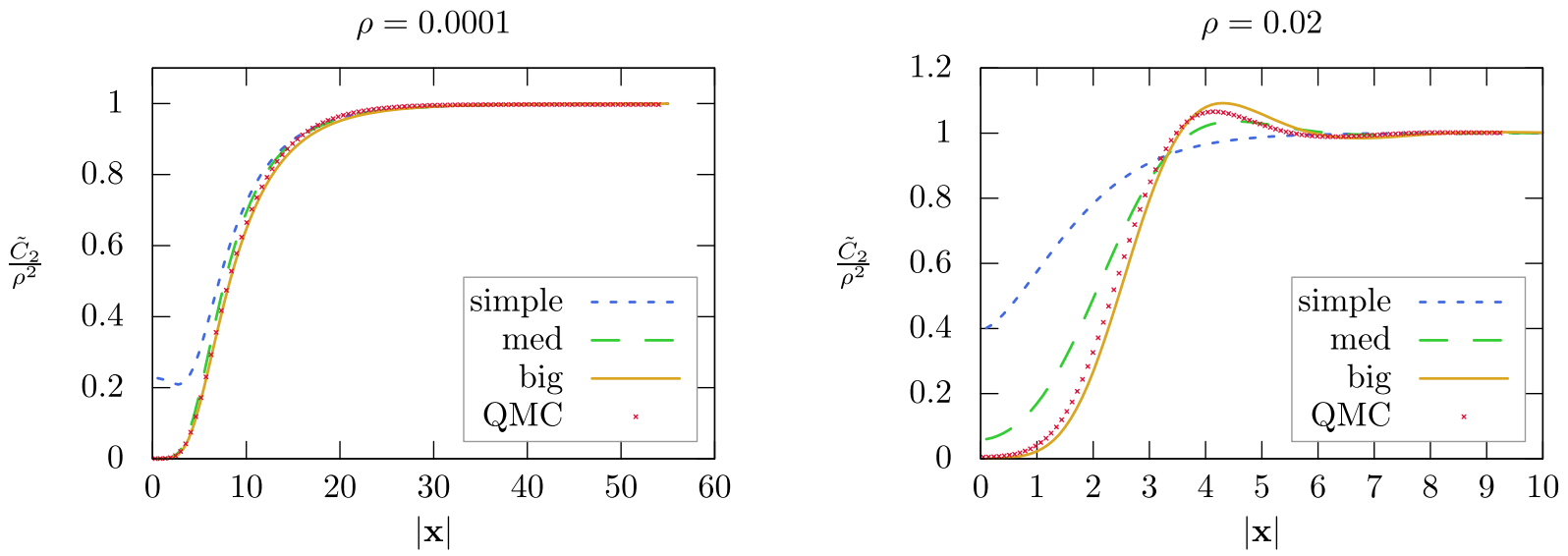

FIG. 5. $\frac{\tilde{C}_{2}}{\rho^{2}}$ for the potential $16 e^{-|\mathbf{x}|}$ at $\rho=0.0001$ (left) and $\rho=0.02$ (right). We compare the predictions of the big, medium, and simple equations to a quantum Monte Carlo simulation.

[1] I. Jauslin (2021), http://ian.jauslin.org/software/simplesolv. 